Diabetologe 2010 - 6:485-485

DOI 10.1007/s11428-010-0625-4

Online publiziert: 13. August 2010

(c) Springer-Verlag 2010

\author{
B. Nowotny \\ Institut für Klinische Diabetologie, Leibniz-Zentrum für Diabetesforschung, \\ Deutsches Diabetes-Zentrum an der Heinrich-Heine-Universität, Düsseldorf
}

\title{
Mechanismen der fettinduzierten Insulinresistenz im Mausmodell
}

leranz und Insulinsekretion (gemessen mittels intraperitonealem Glukosebelastungstest/IPGTT und Insulintoleranztest/ITT) sowie Insulinsensitivität (gemessen mittels hyperinsulinämischem, euglykämischem Clamp) als die WildtypMäuse. Ein solcher Unterschied war nicht zu beobachten, wenn beide Mausstämme mit der konventionellen Diät gefüttert wurden. Im Fettgewebe der TLR2 ${ }^{-/-}$ Mäuse fanden sich kleinere Fettzellen, die eine bessere insulininduzierte Glukoseaufnahme zeigten.

Interessanterweise war die Expression von Entzündungsmarkern in den Fettzellen weitgehend unverändert, lediglich das Chemokin MCP-1 zeigte unter Hochfettdiät in den Wildtyp-Mäusen eine erhöhte Expression, die durch den Knock-out vermindert wurde, und ebenso waren die zirkulierenden Spiegel von MCP- 1 und TNF- $\alpha$ erniedrigt, während die FFA-Spiegel gleich waren. Die $\mathrm{TLR}_{2}{ }^{-1}$-Mäuse verbrauchten etwa 10\% mehr Energie und nahmen auch diese Menge Kalorien mehr als ihre Wildtyp-Verwandten auf, wobei sie eine erhöhte Fettverbrennung aufwiesen, die auch in einer erhöhten Genexpression von Enzymen der Betaoxidation widergespiegelt wurde.

Die Reduktion der fettinduzierten Insulinsensitivität durch den TLR2-Knockout konnte sowohl im Muskel als auch in der Leber nachgewiesen werden, zudem fand sich keine Fetteinlagerung in der Leber. Die Genexpression von Enzymen des Fettstoffwechsels und Markern der Inflammation war jedoch im Vergleich zu gesunden Kontrollen unverändert. Im aus dem Pankreas isolierten Inseln fand sich in vitro eine verbesserte Insulinsekretion auf Glukose ebenso wie eine reduzierte Expression inflammatorischer Marker in den TLR2 ${ }^{-/}$-Mäusen. Auch in Makrophagen, die aus dem Knochenmark der Mäu- se isoliert wurden, fand sich in den TLR2defizienten Mäusen eine geringere Expression von inflammatorischen Markern auf In-vitro-Stimulation mit FFA.

\section{Kommentar}

Insgesamt konnte diese Studie im Mausmodell nachweisen, dass die fehlende Expression eines immunologischen Rezeptors vor einer Insulinresistenz und vermehrten Entzündungsreaktion in verschiedenen Körpergeweben schützt, wobei sich anscheinend lediglich der Aktivitätsstatus der Immunzellen, nicht aber ihre Anzahl ändert. Diese Befunde waren ausgeprägter in weiblichen als in männlichen Mäusen. Ähnliche Ergebnisse konnten bereits für einen weiteren Rezeptorsubtyp der TLR-Familie gezeigt werden. Da es sich hier um einen generellen Knock-out handelt, kann durch die Studie nicht differenziert werden, ob die Defizienz in den Immunzellen oder im Gewebe der entscheidende protektive Faktor ist. Auch bleibt offen, wie die Hochfettdiät/FFA mit dem TLR2 interagiert. Diese Studie liefert aber einen weiteren wichtigen Hinweis darauf, dass es sich bei der FFA-induzierten Insulinresistenz um ein komplexes Geschehen handelt, das sowohl den Fettstoffwechsel als auch das Immunsystem involviert.

\section{Korrespondenzadresse \\ Dr. B. Nowotny}

Institut für Klinische Diabetologie, Leibniz-Zentrum für Diabetesforschung, Deutsches Diabetes-Zentrum an der Heinrich-Heine-Universität Auf'm Hennekamp 65, 40225 Düsseldorf bettina.nowotny@ddz.uni-duesseldorf.de

Interessenkonflikt. Die korrespondierende Autorin weist auf folgende Beziehung hin: Reisekostenübernahme durch Sanofi-Aventis ser Zeit zeigten insbesondere weibliche $\mathrm{TLR}_{2}{ }^{-/-}$-Mäuse eine bessere Glukoseto- 